\title{
Energetic particle injections into the outer cusp during compression events
}

\author{
D. C. Delcourt ${ }^{1}$, H. V. Malova ${ }^{2,5}$, L. M. Zelenyi ${ }^{2}$, J.-A. Sauvaud ${ }^{3}$, T. E. Moore ${ }^{4}$, and M.-C. Fok ${ }^{4}$ \\ ${ }^{1}$ CETP-CNRS, Saint-Maur des Fossés, France \\ ${ }^{2}$ IKI-Space Research Institute, Moscow, Russia \\ ${ }^{3}$ CESR-CNRS, Toulouse, France \\ ${ }^{4}$ NASA-GSFC, Greenbelt, USA \\ ${ }^{5}$ Skobeltsyn Institute of Nuclear Physics, MSU, Moscow, Russia
}

(Received September 9, 2004; Revised January 8, 2005; Accepted January 17, 2005)

\begin{abstract}
We investigate the dynamics of charged particles in the dayside magnetosphere in response to abrupt variations of the solar wind dynamical pressure. Using test particle simulations, we show that the electric field induced by the compression of the frontside magnetosphere may be responsible for prominent energization of plasma sheet ions as well as trapping at high latitudes. We demonstrate that, due to the short-lived character of the magnetic field line reconfiguration (on the time scale of a few minutes), the particle magnetic moment (first adiabatic invariant) may not be conserved during such events. Ions that are initially bouncing from one hemisphere to the other are found to experience nonadiabatic energization up to the hundred of $\mathrm{keV}$ range while being injected into the outer cusp. Such injections involve particles from limited portions of the dayside flux tubes. The energetic particles that are produced in the outer cusp during such events subsequently circulate about the field minimum at high latitudes without intercepting the equatorial plane, thus contributing to the high-energy populations that are observed in this region of space.
\end{abstract}

Key words: Cusp, magnetospheric configuration and dynamics, charged particle motion and acceleration, trapped energetic particles, numerical modeling.

\section{Introduction}

Due to the interaction with the solar wind, the outer cusp in the dayside magnetosphere is a region of weak magnetic field (e.g., Petrinec and Russell, 1995). This topological feature is of particular importance for the dynamics of charged particles since it leads to a mirror force that points away from the equator in the vicinity of the frontside magnetopause; hence, the possible de-trapping of equatorial particles (e.g., Shabansky, 1971). In this region of space, numerical simulations indeed reveal a branching of the particle orbits from mirroring about the equatorial plane to mirroring about the cusp field minimum at high latitudes, possibly followed by escape into the magnetospheric boundary layers (Delcourt and Sauvaud, 1999). On the other hand, recent observations from the POLAR spacecraft provided evidences of high-energy populations in the outer cusp region, with energies ranging from tens of $\mathrm{keV}$ up to hundreds of keV (e.g., Chen et al., 1998). Sheldon et al. (1998) put forward that, at such energies, particles may exhibit closed orbits about the cusp field minimum, rapidly drifting in latitude and azimuth without intercepting the equatorial plane (see, e.g., figure 2 of their study). Different mechanisms have been proposed to explain the build-up of such highenergy populations. Based on the comparison of particle spectra, various studies (e.g., Chang et al., 1998; Trattner et al., 2001) suggested that these populations are accelerated

Copy right(c) The Society of Geomagnetism and Earth, Planetary and Space Sciences (SGEPSS); The Seismological Society of Japan; The Volcanological Society of Japan; The Geodetic Society of Japan; The Japanese Society for Planetary Sciences; TERRAPUB at the bow shock and subsequently transported downstream. At variance with this interpretation, Chen et al. (1998) argued that some local acceleration process in the cusp region is responsible for these energetic populations, an issue that remains both conjectural and controversial (e.g., Sheldon et al., 2003; Trattner et al., 2003). Fritz et al. (2000) suggested that these populations are locally produced by turbulent electric fields and may actually form a significant supply of energetic plasma for the inner magnetosphere.

In this short paper, we examine the impact of compressions of the dayside magnetosphere due to sudden enhancements of the solar wind dynamical pressure. We show that such dynamical reconfigurations of the magnetic field lines may lead to prominent particle energization and injection into the outer cusp. Previous studies already showed that variations of the solar wind dynamical pressure are responsible for injections of energetic particles into the inner magnetosphere (e.g., Li et al., 2003) and actually lead to perceptible changes of the magnetic field at the planet surface (e.g., Le and Russell, 1993). Here, we focus on the effect of the electric field induced by the time-varying magnetic field at high altitudes. We demonstrate that, under the effect of such short-lived electric fields, particles may not conserve their magnetic moment and experience an intense (several tens of $\mathrm{keV}$ and above) nonadiabatic energization which exceeds that expected in the adiabatic case (betatron or Fermitype). The large magnetic moment variations experienced by the particles also lead to high-altitude mirror points and consequent trapping near the cusp field minimum. To set up the framework of this study, we present in Section 2 a 


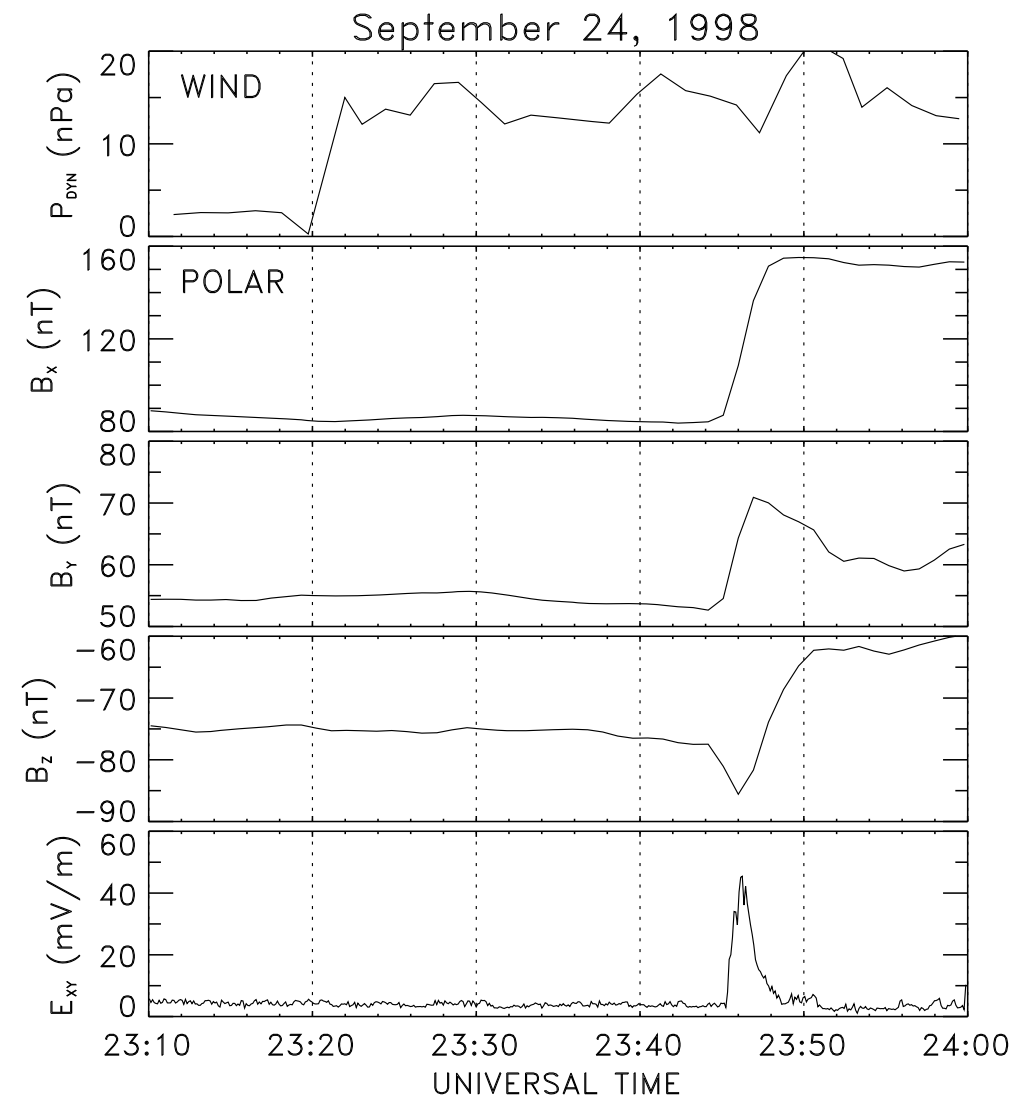

Fig. 1. Plasma and field measurements recorded on September 24, 1998: (from top to bottom) solar wind dynamical pressure at WIND, GSE magnetic field and electric field components at POLAR.

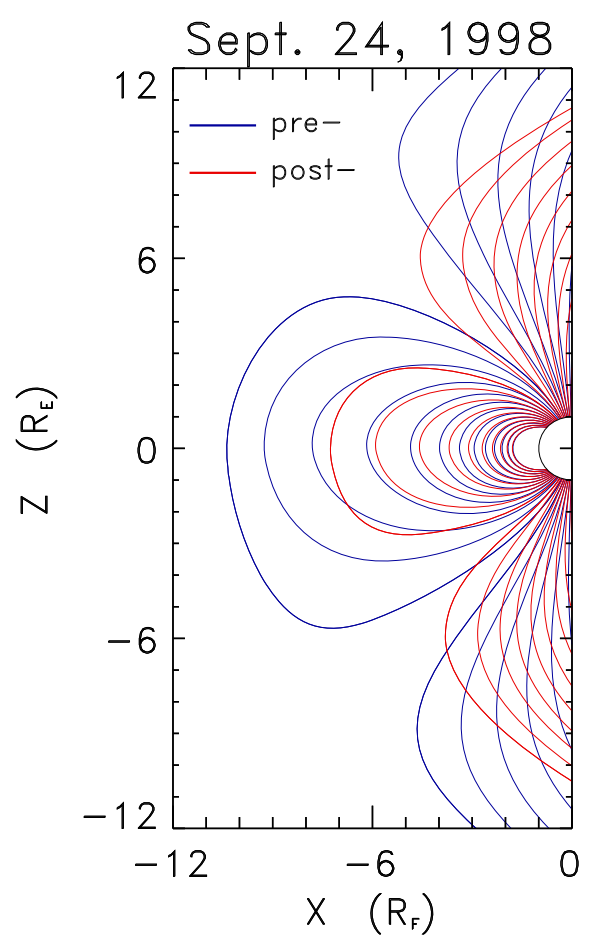

Fig. 2. Configuration of the dayside magnetosphere obtained from the Tsyganenko (1996) model before (blue lines) and after (red lines) the large increase of the solar wind dynamical pressure on September 24, 1998 (see Fig. 1). Note that, in this figure as well as in the following, the $X$ axis points in the antisunward direction and the $Y$ axis, from dusk to dawn. case that exemplifies the compression of the dayside magnetosphere. The numerical model adopted to describe such reconfigurations of the frontside sector is presented in Section 3, whereas the dynamics of charged particles during such events is investigated in Section 4.

\section{Example of Magnetospheric Compression}

Figure 1 shows an example of a large magnetospheric compression that occurred on September 24, 1998. This event was examined in various studies (e.g., Moore et al., 1999; Russell et al., 1999; Strangeway et al., 2000) which in particular put forward a rapid response of the ionosphere with enhanced outflows of low-energy plasma. The top panel of Fig. 1 shows the solar wind dynamical pressure measured by WIND at a distance of $\sim 185 \mathrm{R}_{E}$ upstream of the Earth. In this panel, an abrupt pressure increase (from a few $\mathrm{nPa}$ up to $\sim 14 \mathrm{nPa}$ ) is noticeable near 23:20 UT. This increase is accompanied by large enhancements of the $X$ and $Y$ components of the interplanetary magnetic field (not shown). Some 25 minutes later, an abrupt increase of the magnetic field magnitude was recorded by POLAR, essentially in the $X$ direction (middle panels of Fig. 1). At this time, POLAR was located near apogee over the northern polar cap, at about $8.7 \mathrm{R}_{E}$ geodistance, $69^{\circ}$ magnetic latitude, and 03:40 magnetic local time. The bottom panel of Fig. 1 which shows the electric field recorded onboard POLAR, exhibits a large spike of a few tens of $\mathrm{mV} / \mathrm{m}$ between $\sim 23: 45$ and $\sim 23: 47$ UT. This spike is well correlated with the magnetic field variation, hence likely of inductive na- 
ture.

Some insights into the magnetospheric configuration change that occurred during this event may be obtained from Fig. 2. In a like manner to figure 4 of Russell et al. (1999), this figure shows the magnetic field lines obtained from the Tsyganenko (1996) model using the interplanetary magnetic field (IMF) and solar wind parameters measured at WIND (viz., a solar wind dynamical pressure varying from 2 to $14 \mathrm{nPa}$, an IMF $\mathrm{B}_{Y}$ component varying from 12 to $37 \mathrm{nT}$, an IMF $\mathrm{B}_{Z}$ component varying from -2 to $10 \mathrm{nT}$, and a $D s t$ index of $-43 \mathrm{nT}$ ). It is apparent from Fig. 2 that the large enhancement of the solar wind dynamical pressure displayed in the top panel of Fig. 1 leads to a quite significant compression of the dayside magnetosphere, with a subsolar point that moves from $\sim 10 \mathrm{R}_{E}$ down to $\sim 7 \mathrm{R}_{E}$. Figure 2 also reveals that, after compression, the magnetic field over the northern polar cap is more sunward oriented, consistently with the POLAR measurements in the bottom panels of Fig. 1.

It should be stressed that the September 24, 1998 event is somewhat unusual and is shown here to exemplify the reconfiguration of the dayside magnetosphere under the effect of abrupt solar wind dynamical pressure variations. In Fig. 2, we use the Tsyganenko (1996) model because it allows to take into account the IMF and solar wind control of the magnetosphere using observed parameter values. Here, these parameter values however are somewhat extreme with regard to the Tsyganenko (1996) domain of validity so that the configurations displayed in Fig. 2 should only be considered from a qualitative viewpoint like in the study of Russell et al. (1999). In the following numerical simulations, a more moderate reconfiguration of the dayside magnetosphere will be considered. We will also use a different magnetic field model that allows us to derive the electric field induced by the time-varying magnetic field during compression, which is not possible with Tsyganenko (1996) given the complexity of this latter model.

\section{Modeling of Magnetospheric Compression}

Figure 2 suggests that, within a few minutes, the dayside magnetosphere may be subjected to a prominent configuration change as a result of enhanced solar wind dynamical pressure. Though not as extreme as that portrayed in Figs. 1-2, the frontside magnetopause is routinely subjected to such compressions (e.g., Sibeck, 1990). The reconfiguration of the magnetic field lines during such events leads to an induced electric field which in return may be responsible for particle energization, and it is thus of interest to examine the dynamics of charged particles during such magnetic transitions. Because an analytical description of the electric field that is induced by a time-varying magnetic field (viz., $\mathbf{E}=-\partial \mathbf{A} / \partial t$ where $\mathbf{A}$ is the vector potential) in the complex Tsyganenko (1996) model is not available, we use here a simpler magnetic field model that reproduces the essential features of the time-varying dayside magnetosphere. We adopt an approach similar to that used in the study of Delcourt et al. (1990) where storm-time reconfigurations of the magnetospheric field lines were accounted for by considering a gradual transition within the Mead and Fairfield (1975) model, from a given level of magnetic activity to a less disturbed one. In Delcourt et al. (1990), the magnetic field at a given position $\mathbf{r}$ and time $t$ was given by:

$$
\mathbf{B}(\mathbf{r}, t)=\mathbf{B}_{\text {pre- }}(\mathbf{r})+f(t)\left[\mathbf{B}_{\text {post }-}(\mathbf{r})-\mathbf{B}_{\text {pre- }}(\mathbf{r})\right]
$$

where $\mathbf{B}_{\text {pre- }}$ and $\mathbf{B}_{\text {post- }}$ correspond to initial and final configurations, respectively. Also, in this equation, $f(t)$ is a polynomial of degree 5 that smoothly varies between 0 at $t=0$ and 1 at $t=\tau$ (denoting by $\tau$ the time scale of the magnetic transition; see appendix A of Delcourt et al. (1990)). As for the electric field, it was considered to be the sum of two contributions. One of them is purely induced by the time-varying magnetic field, whereas the other is irrotational and accounts for plasma polarization. As discussed by Heikkila and Pellinen (1977), this latter contribution which is built by free charges is introduced to cancel the parallel component of the electric field obtained from Faraday's law and leads to a redistribution of the electric field in the perpendicular direction (see appendix B of Delcourt et al. (1990)). At a given position $\mathbf{r}$ and time $t$, the electric field was thus obtained as:

$$
E(\mathbf{r}, t)=-\frac{\partial \mathbf{A}(\mathbf{r}, t)}{\partial t}-\nabla \Phi_{\mathrm{pol}}(\mathbf{r}, t)
$$

where $\nabla \Phi_{\text {pol }}$ is the electric potential due to the free charges, and $\mathbf{A}$ is the instantaneous vector potential such that:

$$
\mathbf{A}(\mathbf{r}, t)=\mathbf{A}_{\text {pre- }}(\mathbf{r})+f(t)\left[\mathbf{A}_{\text {post }-}(\mathbf{r})-\mathbf{A}_{\text {pre- }}(\mathbf{r})\right]
$$

where $\mathbf{A}_{\text {pre- }}$ and $\mathbf{A}_{\text {post- }}$ relate to initial and final configurations, respectively.

In the present study, we adopt a calculation technique similar to (1)-(3) but, instead of the Mead and Fairfield (1975) model, we use that of Tsyganenko (1989) which offers an analytical description of the vector potential (hence, the derivation of the induced electric field) and provides a relatively accurate description of the cusp region (e.g., Russell, 2000). In this latter model, the change in the dayside magnetosphere configuration due to compression is accounted for by varying the level of magnetic activity as is illustrated in Fig. 3. In this figure, in view of the measurements displayed in Fig. 1, we assume that the time scale of the transition from one configuration to the other is two minutes. It is apparent from Fig. 3 that, during this transition, the subsolar point moves earthward, from $\sim 11 \mathrm{R}_{E}$ in the initial configuration (blue pattern) down to $\sim 10 \mathrm{R}_{E}$ in the final one (red pattern). Also, the vectors in the left panel of Fig. 3 depict the trajectories of virtual "zero-energy" particles which are only subjected to $\mathbf{E} \times \mathbf{B}$ drift during the magnetic transition. It can be seen that such particles are expelled toward higher latitudes under the effect of a transient induced electric field that reaches several $\mathrm{mV} / \mathrm{m}$ in the outer cusp (bottom right panel). Note that because we consider a moderate reconfiguration of the frontside magnetosphere, this electric field magnitude is significantly smaller than the extreme one displayed in Fig. 1.

\section{Particle Dynamics during Magnetospheric Compression}

To illustrate the impact of the dayside magnetosphere reconfiguration on the charged particle dynamics, Figure 4 

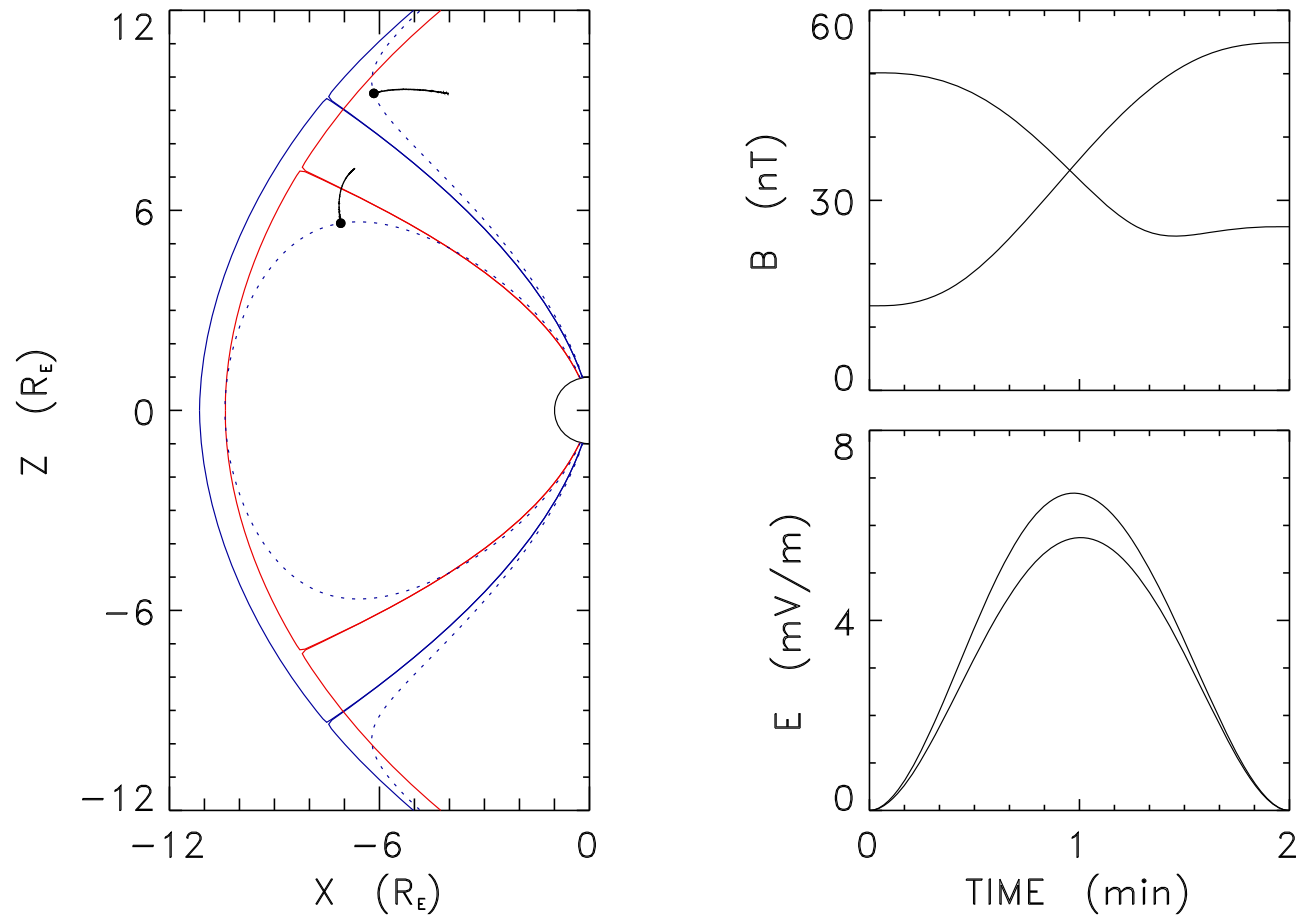

Fig. 3. Model compression of the frontside magnetosphere in the Tsyganenko (1989) model. The left panel shows the magnetopause location before (blue lines) and after (red lines) compression, whereas the vectors indicates the path of virtual "zero-energy" particles during compression. The right panels show the magnetic field and electric field variations encountered along these paths.
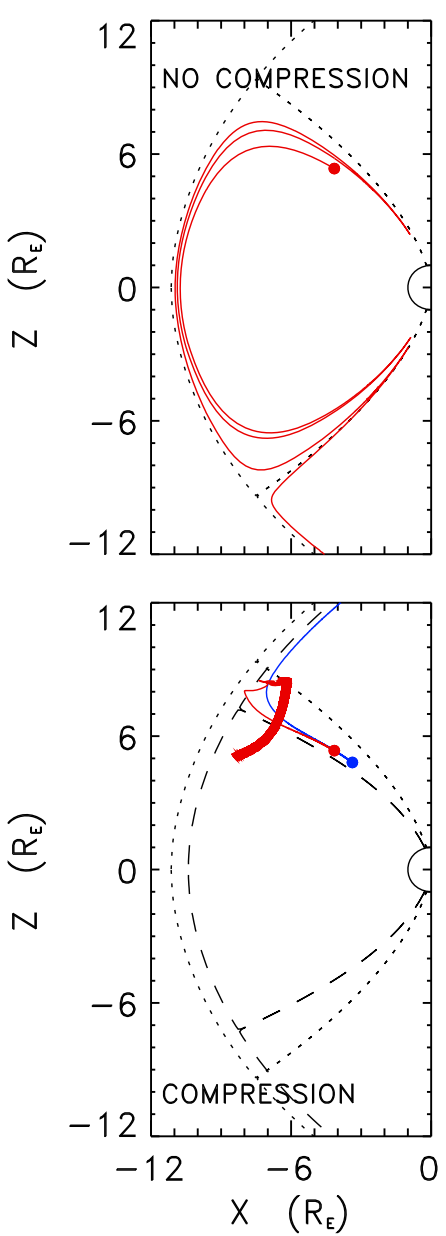
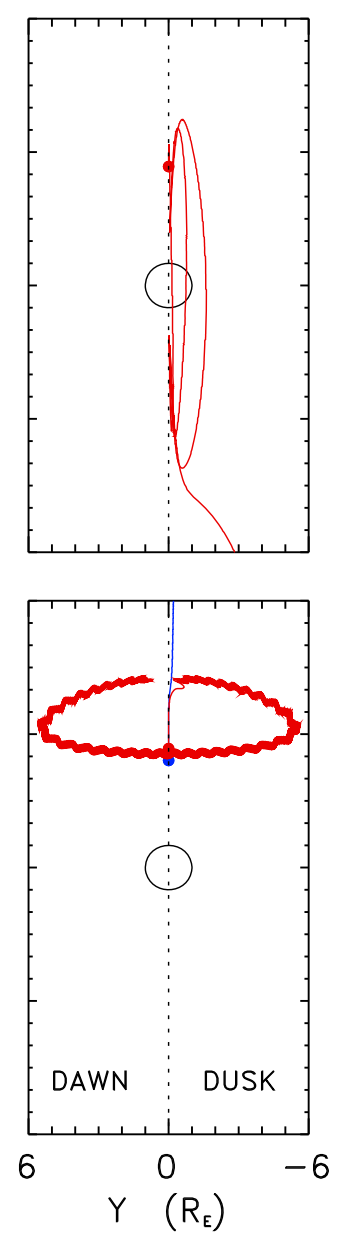
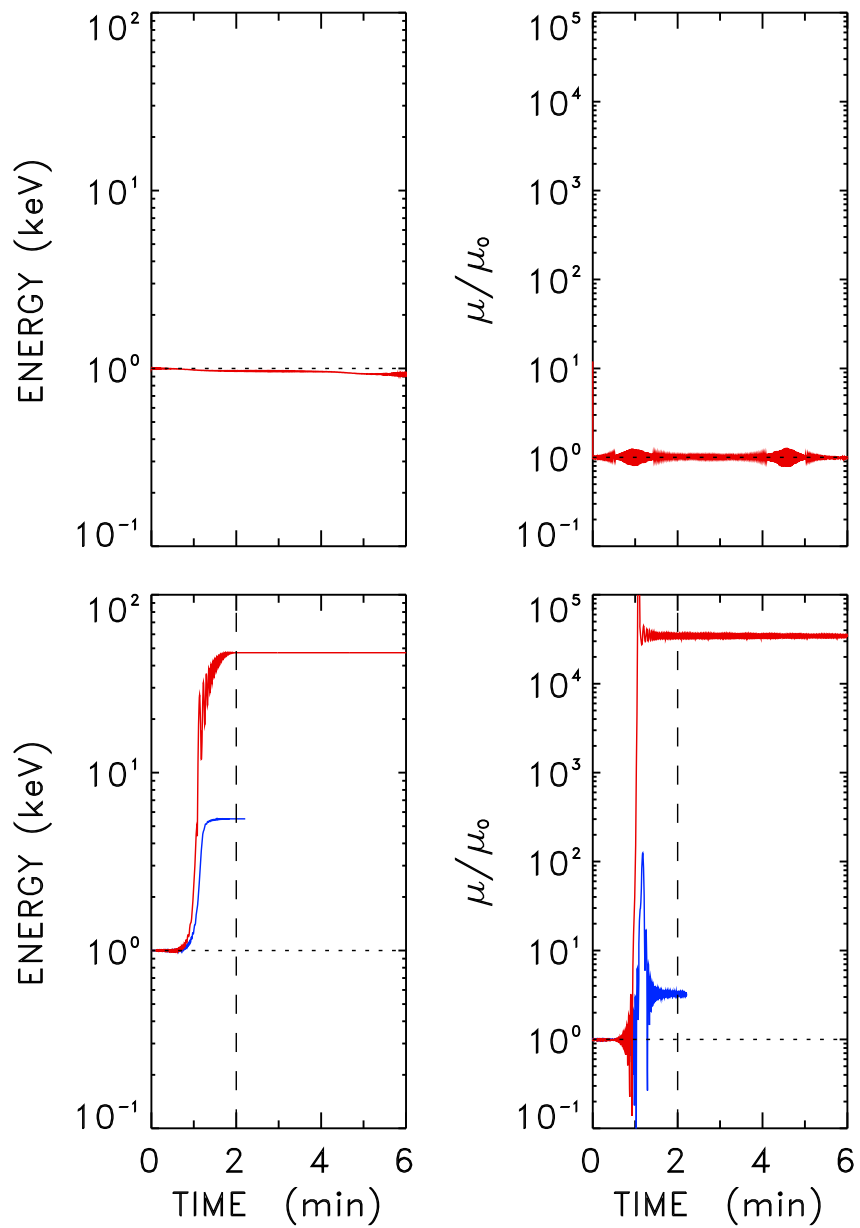

Fig. 4. (top) Model $\mathrm{H}^{+}$orbit in the steady state dayside magnetosphere: (from left to right) trajectory projections in the $X-Z$ and $Y-Z$ planes, energy and magnetic moment (normalized to the initial value) versus time. (bottom) Identical to the top panels but assuming a two-minute compression as in Fig. 3. The orbit coded in blue shows the behavior of a test $\mathrm{H}^{+}$initialized along the same field line but closer to the ionosphere. 

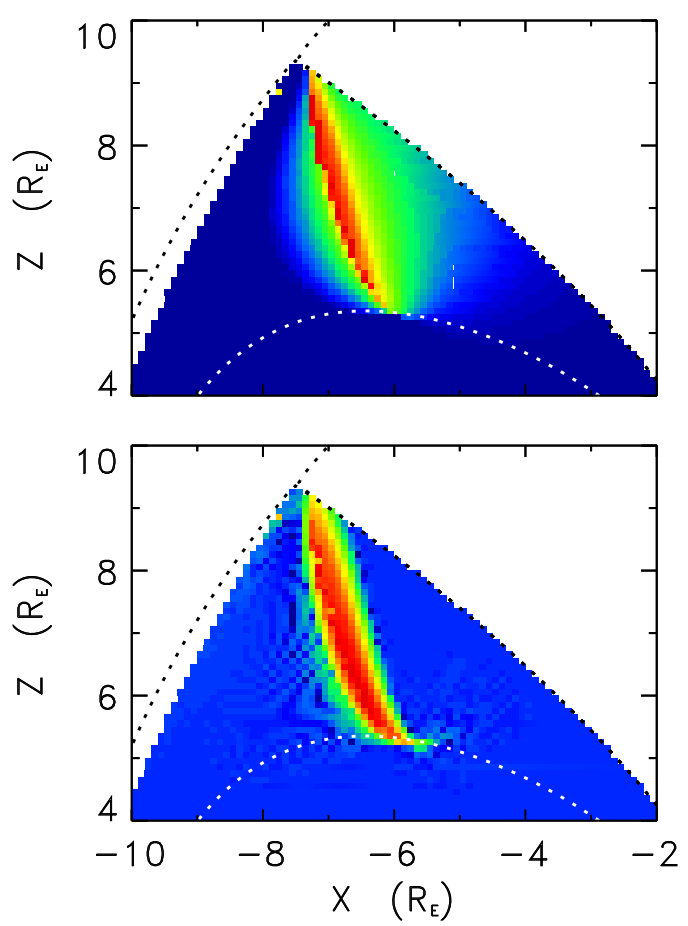
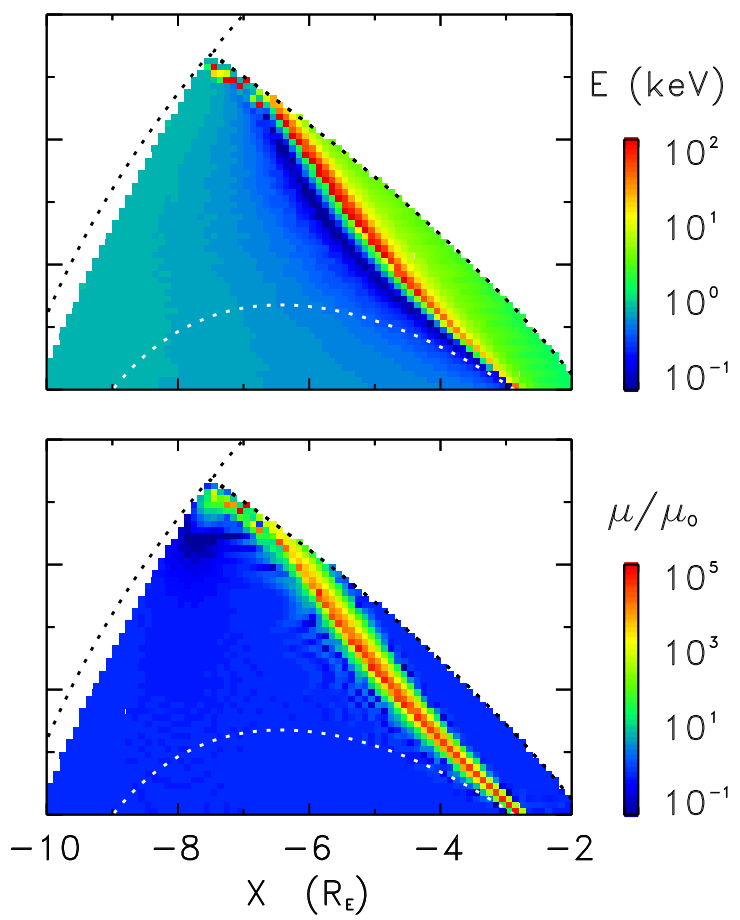

Fig. 5. (top) Post-compression energy and (bottom) magnetic moment (normalized to the initial value) of test $\mathrm{H}^{+}$as a function of initial position in the $X-Z$ plane. Energies and magnetic moments are coded according to the color scales at right. Two distinct initial energies are considered, viz., 100 $\mathrm{eV}$ and $1 \mathrm{keV}$ in the left and right panels, respectively.

shows the trajectory of a test proton both in steady state (top panels) and during a compression event (bottom panels). We assume this test $\mathrm{H}^{+}$to pertain to dayside plasma sheet populations and initialize it in the closed field line region with $1 \mathrm{keV}$ energy and $175^{\circ}$ pitch angle. Not surprisingly, it can be seen in the top panels of Fig. 4 that, in steady state, the test $\mathrm{H}^{+}$bounces back and forth between the two hemispheres, exhibiting mirror points at $\sim 1 \mathrm{R}_{E}$ altitude. This ion simultaneously convects toward higher ILATs under the effect of the $\mathbf{E} \times \mathbf{B}$ drift and it ultimately crosses the boundary between open and closed magnetic field lines (located at $\sim 78.4^{\circ}$ ILAT). It subsequently finds itself onto lobe field lines that extend into the nightside sector over the polar cap and then travels downtail, hence contributing to distant populations (e.g., Seki et al., 1998).

The above steady state behavior contrasts with that obtained in the bottom panels of Fig. 4 where a compression of the dayside magnetosphere identical to that portrayed in Fig. 3 strikes at some point during transport. It can be seen in these bottom panels that, under the effect of the induced electric field, the test $\mathrm{H}^{+}$is rapidly trapped at high latitudes in the outer cusp. There, it executes a closed orbit about the field minimum, which clearly resembles the behavior displayed in Sheldon et al. (1998). The bottom right panels of Fig. 4 reveal that this trapping at high latitudes follows from a prominent magnetic moment enhancement (by more than 4 orders of magnitude), the net energy gain being of the order of $50 \mathrm{keV}$. As a result of the compression, the test $\mathrm{H}^{+}$ thus evolves from a nearly parallel motion along the dayside magnetic field lines to a nearly perpendicular one in the outer cusp where the particle remains trapped. As can be expected from the short-lived (two minutes) character of the magnetic transition considered, these nonadiabatic energization and trapping strongly depend upon the particle position at onset or, equivalently, upon bounce phase. This can be appreciated from the trajectory coded in blue in the bottom panels of Fig. 4 which shows a test $\mathrm{H}^{+}$initialized along the same field line but $1 \mathrm{R}_{E}$ closer to the ionosphere. In this latter case, a different phasing between the frontside compression and the ion motion along the field lines leads to a lesser energization (up to $\sim 6 \mathrm{keV}$ ), the test $\mathrm{H}^{+}$being ultimately ejected toward high latitudes.

It is apparent from Fig. 4 that the nonadiabatic particle transport under the effect of the large transient electric field induced by compression of the frontside magnetosphere provides an effective mechanism for energization and trapping into the outer cusp. This cusp accelerator mechanism may contribute to the high-energy populations observed in this region of space (e.g., Chen et al., 1998). Because of the short-lived character of the induced electric field and because particles have large gyro-periods near the cusp field minimum, their motion is nonadiabatic not only with regard to the second adiabatic invariant (hence, dependence upon bounce phase) but also with regard to the first adiabatic invariant (hence, mirror point elevation and trapping at high latitudes). In essence, the behavior portrayed in Fig. 4 with a small-scale ring current-like structure at high latitudes is similar to that obtained during storm-time reconfiguration of the magnetotail. In this latter case, ions initially bouncing back and forth along magnetic field lines while drifting westward are subjected to a large nonadiabatic energization in the course of convection surges and are subsequently injected into the equatorial ring current (e.g., Delcourt, 2002).

To obtain a more global view of the behavior displayed 
in Fig. 4, we performed systematic $\mathrm{H}^{+}$trajectory calculations considering two distinct initial energies $(100 \mathrm{eV}$ and $1 \mathrm{keV}$ ) and various initial positions in the closed field line region of the dayside magnetosphere. The results of these computations are shown in Fig. 5 which presents the post-compression energy (top panels) and magnetic moment (bottom panels) of the particles as a function of initial position. Figure 5 exhibits several features of interest, namely: as a result of the short-lived compression of the frontside magnetosphere, particles may be subjected to prominent magnetic moment enhancement and energization up to the hundred of keV range. Such energy gains under the effect of the induced electric field significantly exceed those expected from the large-scale convection electric field. Also, because of phasing effects between bouncing motion and the surging electric field, the energetic particles injected into the outer cusp originate from a limited region of space. On either side of this region, ions exhibit weak energy gains and are transported adiabatically (hence, betatron and Fermi-type energization) as evidenced from the negligible magnetic moment variations in the bottom panels of Fig. 5. Not surprisingly, Figure 5 also reveals that the appropriate bounce phase for injection into the outer cusp depends upon the initial energy of the particles, hence the distinct patterns in the left and right panels. Finally, these injections are restricted to the outermost closed field lines, involving particles that are located above $\sim 74^{\circ}$ ILAT (white dotted lines), i.e., within a few degrees from the open-closed field line boundary.

\section{Conclusion}

The numerical simulations performed demonstrate that sudden compressions of the frontside magnetosphere due to enhanced dynamical pressure of the solar wind may lead to a rapid transport of ions from the dayside plasma sheet into the outer cusp. The short-lived electric field induced by the reconfiguration of the magnetic field lines in the outer dayside sector possibly yields ion energization of several tens of keVs in conjunction with large magnetic moment enhancement. The energetic particles thus produced are trapped for some time about the cusp field minimum at high latitudes. They locally exhibit closed orbits with rapid azimuthal drift, hence possibly contributing to the highenergy populations observed in this region of space.

Acknowledgments. We thank F. Mozer, K. Ogilvie, and C. T. Russell for the use of WIND and POLAR data.

\section{References}

Chang, S. W., J. D. Scudder, S. A. Fuselier, J. F. Fennell, K. J. Trattner, J. S. Pickett, H. E. Spence, J. D. Menietti, W. K. Peterson, R. P. Lepping, and R. Friedel, Cusp energetic ions: A bow shock source, Geophys. Res. Lett., 25, 3729, 1998.

Chen, J., T. A. Fritz, R. B. Sheldon, H. E. Spence, W. N. Speldvijk, J. F. Fennell, S. Livi, C. T. Russell, J. S. Pickett, and D. A. Gurnett, Cusp energetic particle events: Implications for a major acceleration region of the magnetosphere, J. Geophys. Res., 103, 69, 1998.
Delcourt, D. C., Particle acceleration by inductive electric fields in the inner magnetosphere, J. Atm. Solar Terr. Phys., 64, 551, 2002.

Delcourt, D. C. and J.-A. Sauvaud, Population of the cusp and boundary layers by energetic (hundreds of keV) equatorial particles, J. Geophys. Res., 104, 22,635, 1999.

Delcourt, D. C., J.-A. Sauvaud, and A. Pedersen, Dynamics of singleparticle orbits during substorm expansion phase, J. Geophys. Res., 95, 20,853, 1990.

Fritz, T. A., J. Chen, and R. B. Sheldon, The role of the cusp as a source for magnetospheric particles: A new paradigm?, Adv. Space Res., 25, 1445 , 2000.

Heikkila, W. J. and R. J. Pellinen, Localized induced electric field within the magnetotail, J. Geophys. Res., 82, 1610, 1977.

Le, G. and C. T. Russell, Effect of sudden solar wind dynamic pressure changes at subauroral latitudes: Time rate of change of magnetic field, Geophys. Res. Lett., 20, 1, 1993.

Li, X., D. N. Baker, S. Elkington, M. Temerin, G. D. Reeves, R. D. Belian, J. B. Blake, H. J. Singer, W. Peria, and G. Parks, Energetic particle injections in the inner magnetosphere as a response to an interplanetary shock, J. Atm. Solar Terr. Phys., 65, 233, 2003.

Mead, G. D. and D. H. Fairfield, A quantitative magnetospheric model derived from spacecraft magnetometer data, J. Geophys. Res., 80, 523, 1975.

Moore, T. E., W. K. Peterson, C. T. Russell, M. O. Chandler, M. R. Collier, H. L. Collin, P. D. Craven, R. Fitzenreiter, B. L. Giles, and C. J. Pollock, Ionospheric mass ejection in response to a CME, Geophys. Res. Lett., 26, 2339, 1999.

Petrinec, S. M. and C. T. Russell, An examination of the effect of dipole tilt angle and cusp regions on the shape of the dayside magnetopause, $J$. Geophys. Res., 100, 9559, 1995.

Russell, C. T., The polar cusp, Adv. Space Res., 25, 1413, 2000.

Russell, C. T., X. W. Zhou, P. J. Chi, H. Kawano, T. E. Moore, W. K. Peterson, J. B. Cladis, and H. J. Singer, Sudden compression of the outer magnetosphere associated with an ionospheric mass ejection, Geophys. Res. Lett., 26, 2343, 1999.

Seki, K., M. Hirahara, T. Terasawa, T. Mukai, Y. Saito, S. Machida, T. Yamamoto, and S. Kokubun, Statistical properties and possible supply mechanisms of tailward cold $\mathrm{O}^{+}$beams in the lobe/mantle regions, $J$. Geophys. Res., 103, 4477, 1998.

Shabansky, V. P., Some processes in the magnetosphere, Space Sci. Rev., 12, 299, 1971.

Sheldon, R. B., H. E. Spence, J. D. Sullivan, T. A. Fritz, and J. Chen, The discovery of trapped energetic electrons in the outer cusp, Geophys. Res. Lett., 25, 1825, 1998.

Sheldon, R. B, J. Chen, and T. A. Fritz, Comment on "Origins of energetic ions in the cusp" by K. J. Trattner et al., J. Geophys. Res., 108, 1302, doi:10.1029/2002JA009575, 2003.

Sibeck, D. G., A model for the transient magnetospheric response to sudden solar wind dynamic pressure variations, J. Geophys. Res., 95, 3755, 1990.

Strangeway, R. J., C. T. Russell, C. W. Carlson, J. P. McFadden, R. E. Ergun, M. Temerin, D. M. Klumpar, W. K. Peterson, and T. E. Moore, Cusp field-aligned currents and ion outflows, J. Geophys. Res., 105, 21,129, 2000.

Trattner, K. J., S. A. Fuselier, W. K. Peterson, S.-W. Chang, R. Friedel, and M. R. Aellig, Origins of energetic ions in the cusp, J. Geophys. Res., 106, 5967, 2001.

Trattner, K. J., S. A. Fuselier, W. K. Peterson, S.-W. Chang, R. Friedel, and M. R. Aellig, Reply to comment on "Origins of energetic ions in the cusp" by R. Sheldon, J. Chen, and T. A. Fritz, J. Geophys. Res., 108, 1303, doi:10.1029/2002JA009781, 2003.

Tsyganenko, N. A., Magnetospheric magnetic field model with a warped tail current sheet, Planet. Space Sci., 37, 5, 1989.

Tsyganenko, N. A., Effects of the solar wind conditions on the global magnetospheric configuration as deduced from data-based field models, in European Space Agency Publication ESA SP, 389, 181, 1996.

D. C. Delcourt (e-mail: dominique.delcourt@cetp.ipsl.fr), H. V. Malova, L. M. Zelenyi, J.-A. Sauvaud, T. E. Moore, and M.-C. Fok 